The Egyptian Journal of Hospital Medicine (Oct. 2011) Vol., 45: 547 - 553

\title{
Maternal obesity and its adverse impact on labor outcome
}

Inas Mahmoud Hamdy

Department of Obstetrics \&Gynacology faculty of medicine, Al Azhar University Hospital

Abstract:

Objective: to study the impact of maternal obesity on the outcome of labour. Setting: Al zahraa University hospital.Design: prospective randomized control study.Patients: a total of (80) pregnant women were included in this study from April 2009 to March 2010. Patients and Methods:According to the BMI the patients were classified into two groups. Group (I): (Control Group):Included (30) patients with (body mass index) (BMI) ranging from 20 to 25 $\mathrm{kg} / \mathrm{m}^{2}$.Group (II): (Obese Group): Included (50) patients with (BMI) ranging from 25 to $30 \mathrm{~kg} / \mathrm{m}^{2}$. Each patient were subjected to full clinical examination (General) Abdominal and pelvic) U/S pelviabdominal examination that is to confirm the inclusion and exclusion criteria of the study. Results : A significant differences was noticed between 2 groups as regard the BMI. The current study showed significant difference in cases subjected to induction of labour with pvalue $<0.001$. Also significant difference between control group and obese group regarding failed induction, failure to progress $1^{\text {st }}$ stage, $2^{\text {nd }}$ stage, shoulder dystocia with $\mathrm{p}$-value < 0.01.Case of C.S showed the highest significant diff. with P-value $<0.0001$.In the present study no sig. diff. between cases of both groups as regard $3^{\text {rd }}$ stage complication, $4^{\text {th }}$ stage $(\mathrm{PPH})$ and blood transfusion with P-value 0.658, 0.684 and 0.658 respectively. A high significant diff. between the two groups as regard case of macrosomia, Apgar score $<71^{\text {st }}$ minute, and birth injury with p-value $<0.01$. A significant diff. were showed between the cases of both groups as regard the incubation with p-value $>0.05$. No significant diff. were noticed between the 2 groups as regard the cases with Apgar score $<7$ at 5 minutes with p-value 0.06. A positive correlation coefficient between the BMI (25-29), (29-33) kg/m² with the case of induction of labour, failure to progress $1^{\text {st }}$ stage, shoulder dystoca, cesarean section and fetal low Apgar score. While a negative correlation was recorded between the BMI of the control group and all the adverse outcomes. Conclusion:Obesity of the gravid women is a sensitive predictor of the adverse outcome during pregnancy, labour, and post partum. Researches are needed into effective, applicable and acceptable community -based program for obese women planning a pregnancy.

\section{Key words: Maternal obesity. labor}


Maternal obesity and its adverse impact on labor outcome

\section{Introduction:}

The World Health Organization (WHO) describes obesity as: one of the most blatantly visible, yet most neglected, publichealth problems that threatens to overwhelm both more and less developed countries'. A recent study showed that 1 in 5 women booking for antenatal care in 2002-2004 were obese. The increased risk of complications in obese women during pregnancy and delivery coupled with the rising epidemic of obesity among women emphasise the need for the specialists involved in treating obese women to be aware of the risks and complications and their management . (Kanagalingam et al 2005) The obesity-related, adversepregnancy outcomes with a brief outline of the possible physiological mechanisms involved, followed by a discussion of the best practise in managing obese mothers from pregravid to postpartum and their effectiveness in reducing the risk of the obesity-related, adverse outcomes in pregnancy . (Castro et al., 2002) . The study of (Stekkinger et al.,2009) reported the effect of maternal obesity on pregnancy complications with minimal confounding bias. The study supports existing evidence that woman with a BMI $>30 \mathrm{~kg} / \mathrm{m}^{2}$ have a 1-2-fold higher risk of cesarean section. The researchers advocate that this may be an effect of the increased rate of large for gestational age infants leading to disproportion during labour .Controversy exists regarding maternal obesity as a risk factor for shoulder dystocia. It has been argued this association could be related to the correlation of obesity with diabetes mellitus. A case control study by (Robinson et al.,2003) showed that the strongest predictors of shoulder dystocia are related to fetal macrosomia. Furthermore, they found that for obese non-diabetic women carrying fetuses whose weights are estimated to be within normal limits, there In tin Increased risk of shoulder dystocia. Therefore, for obese women, the predictors of shoulder dystocia are similar to those of non-obese women. (Sheiner et al.,2004). has shown that obese women tend to have higher rates of postpartum hemorrhage; the increased incidence of cesarean sections among obese women has been implicated as a causal factor. However, (Usha et al., 2005)showed the increased rate of cesarean section might not be the only factor influencing the blood loss in this group. Obese women who had a vaginal delivery had a greater than $500 \mathrm{ml}$ blood loss compared to those with a BMI of $20-30 \mathrm{~kg} / \mathrm{m}^{2}$. In the study of (Heslehurst et $a l ., 2010)$ proved that macrosomia has been associated with multiple factors, including maternal age and weight. However, increased maternal pre-pregnancy weight and decreased pre-pregnancy insulin sensitivity are strongly correlated with fetal growth, in particular fat mass at birth. During pregnancy, 2-10 kg of fat is stored in women's bodies. About $14-20 \%$ of women are unable to lose this weight, on average gaining $5 \mathrm{~kg}$ or more post- 
pregnancy. However, in obese and overweight women, it remains to be determined whether reduced gestational weight gain can reduce the number of complications during pregnancy and delivery.

Aim of the work: This prospective study aiming to study the impact of maternal obesity on the outcome of labour (maternal and fetal).

\section{Patients and Methods :This is a} prospective study was conducted on a total of 80 pregnant women admitted at $\mathrm{Al}$ zahraa university Hospital for delivery from April 2009 to March 2010 .For each patient: Full history taking including age, parity, calculated gestational age, past hist. to exclude any disease could affect body weight. Full clinical examination. The clinical examination included :Blood pressure, chest, heart, abdominal examination for fundal level, Fundal Grip, and pelvic examination. According to the body mass index the patients were divided into two groups :Group I: included (30) patients with BMI $<25 \mathrm{~kg} / \mathrm{m}^{2}$ and $>20 \mathrm{~kg}$ $/ \mathrm{m}^{2}$ they served as control group.Group II: included (50) patients with BMI $<30 \mathrm{~kg} / \mathrm{m}^{2}$ who served as study group.

Inclusion criteria: Single pregnancy Cephalic presentation, Gestational Age at term - No previous Cesarean Section Placenta fundal - Intact membrane .

\section{Exclusion criteria:}

- Any disease could affect the maternal body weight (Hypertension, diabetes mellitus, etc......).

- Any factor could indicate C.S (malpresentation, placenta previa, previous C.S,etc.....).

- Any factor affect the progress of labour (malposition, inadequate pelvis,etc .....).

- For both groups of patients: The full history and clinical examination helping to confirm the inclusion and exclusion criteria also pelviabdominal U/S were performed for each patient. The BMI were calculated to classify the patients group according to its values.

\section{Laboratory investigations:}

- Blood sugar test.

- Complete blood picture.

- Blood group and RH-factor.

A partogram with electronic fetal monitoring were applied for each case during labour to study the progress of labour and to find out any protraction or failure to progress during $1^{\text {st }}$ stage, $2^{\text {nd }}$ stage, select the patient for C.S. The output of the study were tabulated and analysed statistically. They included. Induction of labour, failure to progress $1^{\text {st }}$ stage, $2^{\text {nd }}$ stage and $3^{\text {rd }}$ stage complication (postpartum $\mathrm{Hg}$ ).

\section{The statistical analysis:}

Dat Data were collected coded and analysis using SPSS, both T test and person's correlation were applied. p-value $>0.05=$ non significant $\mathrm{P}$-value $<0.05=$ significant p-value $<0.01=$ Highly significant

\section{Results:}

Table (I): showed no significant difference between the 2 groups regarding age and parity. A significant difference was noticed between 2 groups as regard the BMI.

Table II: Showed significant difference in cases subjected to induction of labour ( $\mathrm{p}<$ 0.001 ). Also significant increase in the control group than obese group as regard 
failed induction, failure to progress $1^{\text {st }}$ stage, $2^{\text {nd }}$ stage and shoulder dystocia $(\mathrm{p}<$ 0.01).Cases of C.S showed significant increase in obese group when compared to control group $(\mathrm{P}<0.0001$ ). Also table II showed no sig. diff. between cases of both groups as regard $3^{\text {rd }}$ stage complication, $4^{\text {th }}$ stage (PPH) and blood transfusion with Pvalue $0.658,0.684$ and 0.658 respectively. Table III showed significant increase in the obese group than in control group as regard macrosomnia, Apgar score $<71^{\text {st }} \mathrm{mm}$. and birth injury. ( $\mathrm{p}<0.01)$. A significant increase were showed in cases of obese group than control group as regard cases of incubation $(p<0.05)$. No significant diff. were noticed between the 2 groups as regard the cases with Apgar score $<7$ at 5 minutes .Table IV showed a positive correlation between the BMI $25-29,29-33 \mathrm{~kg} / \mathrm{m}^{2}$ with the cases of induction of labour, failure to progress $1^{\text {st }}$ stage, shoulder dystocia, cesarean section and fetal low Apgar score. While Negative correlation was recorded between the BMI of the control group and all the previous items.

Table (I): Shows patients data in both groups (mean \pm S.D).

\begin{tabular}{|l|c|c|c|}
\hline \multicolumn{1}{|c|}{ Item } & $\begin{array}{c}\text { Control G } \\
\mathbf{n = 3 0}\end{array}$ & $\begin{array}{c}\text { Obese G } \\
\mathbf{n = 5 0}\end{array}$ & P value \\
\hline Age & $31.1 \pm 2.4$ & $30.9 \pm 1.1$ & NS \\
\hline Parity & $2.1 \pm 1.1$ & $2.2 \pm 1.3$ & NS \\
\hline B.M.I & $21 . \pm 1.2$ & $29.1 \pm 0.8$ & H.S \\
\hline
\end{tabular}

p-value $>0.05=$ non significant $\mathrm{P}$-value $<0.05=$ significant p-value $<0.01=$ Highly significant

Table (II): Shows comparison between adverse parturition outcome in both groups.

\begin{tabular}{|l|l|l|l|l|l|}
\hline \multicolumn{1}{|c|}{ Item } & \multicolumn{1}{|c|}{ Control G( N=30 ) } & Obese G( N=50 ) & OR & \multicolumn{1}{|c|}{ 95\% CL } & p-value \\
\hline Induction of labour & $8.7 \%$ & $18.3 \%$ & 2.3 & $2.1-2.6$ & 0.001 \\
\hline Failed induction & $1.6 \%$ & $6 \%$ & 4.0 & $3.2-4.9$ & 0.01 \\
\hline $\begin{array}{l}\text { Failure to progress(F.P) } \\
\left(1^{\text {st }} \text { stage }\right)\end{array}$ & $5.9 \%$ & $9.2 \%$ & 1.6 & $1.3-1.9$ & 0.01 \\
\hline (F.P)2 ${ }^{\text {nd }}$ stage & $4.3 \%$ & $8.1 \%$ & 1.8 & $1.4-2.2$ & 0.01 \\
\hline Shoulder dystocia & $3.9 \%$ & $7.8 \%$ & 1.5 & $1.1-1.9$ & 0.01 \\
\hline Cesarean section & $7.2 \%$ & $21.8 \%$ & 3.2 & $2.7-3.7$ & 0.0001 \\
\hline $4^{\text {th }}$ stage (PPH) & $0.4 \%$ & $0.6 \%$ & 1.0 & $0.5-2.1$ & 0.684 \\
\hline Blood transfusion & $3.2 \%$ & $4.0 \%$ & 1.0 & $0.8-1.3$ & 0.658 \\
\hline
\end{tabular}

Table (III): Shows comparison between adverse fetal outcome in both groups.

\begin{tabular}{|l|l|l|l|l|l|}
\hline \multicolumn{1}{|c|}{ Item } & \multicolumn{1}{|c|}{ Control G( N=30 ) } & Obese G( N=50 ) & OR & 95\% CL & p-value \\
\hline Macrosomia & $1.9 \%$ & $5.2 \%$ & 1.6 & $1.2-2.1$ & 0.01 \\
\hline $\begin{array}{l}\text { Apgar score }<71^{\text {st }} \\
\text { minute }\end{array}$ & $2.1 \%$ & $6.9 \%$ & 3.0 & $2.1-3.9$ & 0.01 \\
\hline $\begin{array}{l}\text { Apgar score }<75 \\
\text { minute }\end{array}$ & $1.9 \%$ & $2.3 \%$ & 1.3 & $0.9-1.7$ & 0.06 \\
\hline Incubation & $0.9 \%$ & $2.8 \%$ & 1.4 & $1.1-1.7$ & 0.05 \\
\hline Birth injury & $1.7 \%$ & $4.9 \%$ & 1.8 & $1.3-2.3$ & 0.01 \\
\hline
\end{tabular}

$\mathrm{P}$ value $<0.05 \mathrm{~S}, \mathrm{P}$. value $<0.01$ H.S 
Table (IV): Shows correlation coefficient of BMI to the adverse maternal and fetal outcome.

\begin{tabular}{|l|c|c|c|}
\hline \multicolumn{1}{|c|}{ Item } & $\begin{array}{c}\text { BMI } \\
\mathbf{2 5 - 2 9}\end{array}$ & $\begin{array}{c}\text { BMI } \\
\mathbf{2 9 - 3 3}\end{array}$ & Control G \\
\hline Induction of labour & $0.831^{* *}$ & $0.652^{* * *}$ & $0.942^{*}$ \\
\hline $1^{\text {st }}$ stage failure to progress (FTP) & $0.851^{* *}$ & $0.683^{* * *}$ & $0.951^{*}$ \\
\hline $2^{\text {nd }}$ stage FTP & $0.838^{* *}$ & $0.654^{* * *}$ & $0.981^{*}$ \\
\hline Shoulder dystocia & $0.633^{* * *}$ & $0.611^{* * *}$ & $0.982^{*}$ \\
\hline Cesarean section & $0821^{* *}$ & $0.815^{* *}$ & $0.922^{*}$ \\
\hline Low apgar score & $0.825^{* *}$ & $0.638^{* * *}$ & $0.916^{*}$ \\
\hline
\end{tabular}

* statistically non significant with - ve correlation.

$* * *$ statically significant with + ve correlation.

\section{Discussion :}

Nuthalapaty study proved that the Obese women have increased of labour induction by 1.1-2.2 fold. This finding is coincidence to the result of this current study which revealed a significant increase in cases of induction among obese pregnant women comparing to control group with (P-value < 0.001).Maternal obesity is an independent risk factor for CS this statement come in agreement with the outcome of the present study which showed a significant increase in cases of CS among obese group when compared with control group with( $\mathrm{p}$-value $<0.001)$. Also IQ proved in their study that the CS rate for obese women was over $20 \%$ compared to nearer $10 \%$ for normal weight women. The study of Andreasen \&Chauhan proved that pregnant women with BMI > $30 \mathrm{~kg} / \mathrm{m}^{2}$ have a 3 fold higher incidence in CS.This finding is also found in the present study which showed a statistical significant increase and positive correlation between the BMI over 25-33 and the rate of CS.In the present study there was a significant difference in the cases of shoulder dystocia for the obese group than in the control group with (p-value $<0.01$ ). These findings were similar to the study of (Heslehurst $e t$ al., 2010) which concluded that shoulder dystocia complicated about $2.1 \%$ of obese mothers . Case report study by Baxley and Robinson, they found that for obese non diabetic mothers obesity is a predictor for shoulder dystocia.This is coincidence with the outcome of the current study which found that the BMI $>30 \mathrm{~kg} / \mathrm{m}^{2}$ has a highly significant difference and appositive correlation with the shoulder dystocia.In support to the study of Watkins, Cedergren and Catalano they, found that obese patients have higher incidence of failure to progress during labour than non obese. This out put were similar to the results of the present study which staled that failure to progress during labour ( $1^{\text {st }}$ stage and $2^{\text {nd }}$ stage $)$ were associated with obese group when compared to control group with ( $\mathrm{p}$-value $<0.001$ ). Nalhalapaty, Walker and Galtier contradict to the present study found no significant diff. between obese group and non obese (control group) as regard the post partum haemorhage. This finding could be explained by the difference in patients quality or delivery circumstances. Recently Abrams and Olson proved that the associated between low Apgar score for fetus of obese mothers are of high incidence this observations were in agreement with the output of the current study which revealed significantincrease in the (low Apgar score especially in the $1^{\mathrm{s}}$ minutes) in fetus of obese mothers when compared to control group with( $\mathrm{p}$-value $<0.01)$.

Conclusion:Obesity of the gravid women is a sensitive predictor of the adverse 
outcome during pregnancy, labour, and post partum. Researches are needed into effective, applicable and acceptable

\section{References:}

1. Abrams B, Altman SL, Pickett KE. (2000 ).Pregnancy weight gain: still controversial. Am J Gin Nutr; 71 (Suppl): 1233S-1241S

2. Andreasen KR, Anderen ML, Schantz AL.( 2004). Obesity and pregnancy. Acta Obstet Gynecol Scand; 83: 1022-1029. doi:10.1111/j.0001-6349.2004.00624.

3. Baxley EG, Gobbo RW.( 2004).Shoulder dystocia. Am Fam Phys; 69: 1707-1714

4. Castro L, Avina R. (2002). Maternal obesity and pregnancy outcomes. Curr Opin Obstet Gynecol; 14: 601-606.

5. Catalano PM, Kirwan JP, Haugel-de Mouzon S, King J. (2003).Gestarional diabetes and insulin resistance: role in short- and long-term implications for mother and fetus. J Nutr; 33(Suppl 2): 16745-16835.

6. Cedergren MI. (2004). Maternal morbid obesity and the risk of adverse pregnancy outcome. Vyobstet Gynecol; 103: 219-224.

7. Chauhan SP, Magann EF, Carroll CS, Barrilleaux PS, Scardo JA, Martin Jr JN. (2001).Mode of delivery for the morbidly obese with prior cesarean delivery: vaginal versus repeat cesarean section. Am J Obstet Gynecol; 185: 349-354

8. Galtier-Dereure F, Boegner C, Bringer J.( 2000).Obesity and pregnancy: complications and cost. Am J Clin Nutr; 71 1242S-1248S .

9. Heslehurst N, Rankin J, Wilkinson JR, (2010).Teesside Univ, UK; Newcastle Univ, UK; Durham Univ Queen's Campus UK. Int J Obes 34:420-428.

10. Kanagalingam MG, Forouhi NG, Greer IA, Sattar N. (2005). Changes in booking body mass $\mathrm{Y}^{\wedge}$ index over a decade: retrospective analysis from a Glasgow Maternity

11. Hospital. Br J Obstet Gynaecol; 112: 14311433. community -based program for obese women planning a pregnancy.

12. Lintsen AM, Pasker-de Jong PC, de Boer EJ (2005).Effects of subfertility cause, smoking and body weight on the success rate of H/F. Hum Reprod; 20: 1867-1875.

13. Nuthalapaty FS, Rouse DJ. (2004). The impact of obesity on obstetrical practice and outcome. of Clin Obstet Gynecol; 47: 898913

14. Olson CM, Strawderman MS, Reed RG. (2004). Efficacy of an intervention to prevent excessive gestational weight gain. Am J Obstet Gynecol; 191: 530-536

15. Robinson H, Tkatch S, Mayes DC, Bott N, Okun N. (2003). Is maternal obesity a predictor of shoulder dystocia? Obstet Gynecol; 101: 24-27.

16. Sheiner E, Levy A, Menes TS, Silverberg D, Katz M, Mazor M. (2004).Maternal obesity as an independent risk factor for caesarean delivery. Paediatr Perinat Epidemiol; 18: 196-201

17. Stekkinger E, Zandstra M, Peeters LLH, (2009) Univ Hosp Groningen , the Netherlands; Univ Hosp Maastricht . the Netherlands Obest Gynecol/114:1076-1084

18. Usha KTS, Hemmadi S, Bethel J, Evans J. (2005). Outcome of pregnancy in a woman with an increased body mass index. $\mathrm{Br} \mathrm{J}$ Obstet Gynaecol; 112: 768-772.

19. Walker LO, Sterling BS, Timmerman GM.( 2005). Retention of pregnancy-related weight in the early postpartum period; implications for women's health services.-J Obstet Gynecol Neonatal Nurs; 34: 418-27.

20. Watkins ML, Rasmussen SA, Honein MA, Botto LD, Moore CA. (2003). Maternal obesity and risk for birth defects. Pediatrics; 111: 1152-1158. 


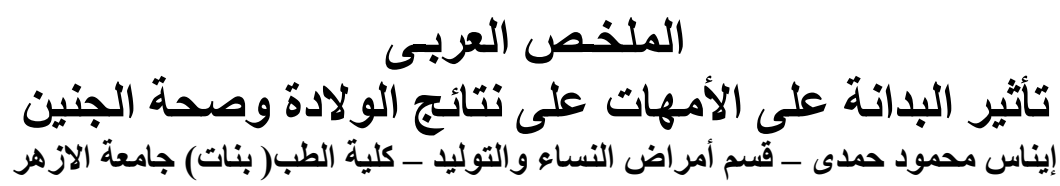

تعتبر منظمة الصحة العالمية أن البدانة و السمنة أصبحت مرض مستقل بذاته دون أى مشاكل مرضية , كما

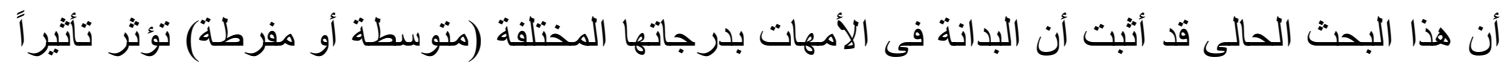

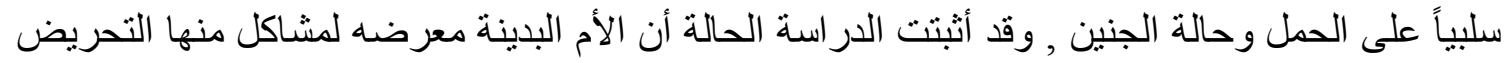

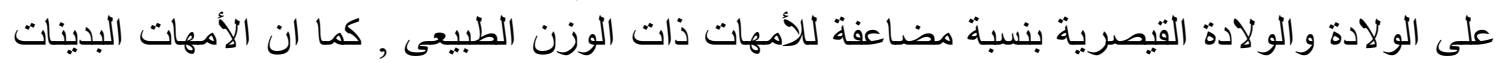

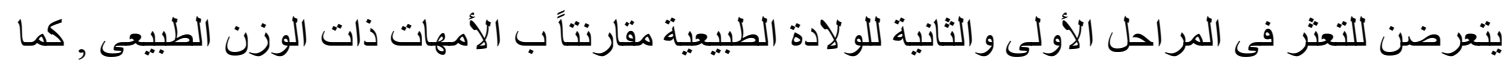

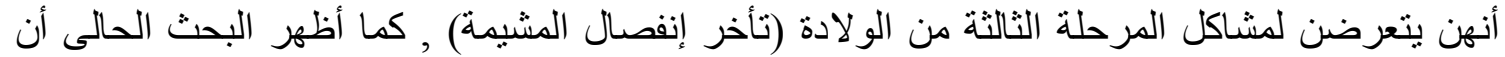

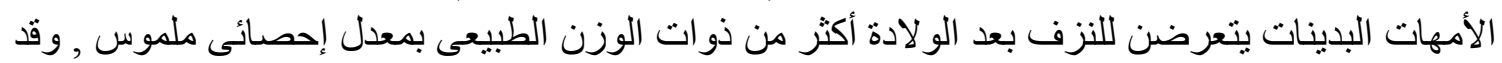

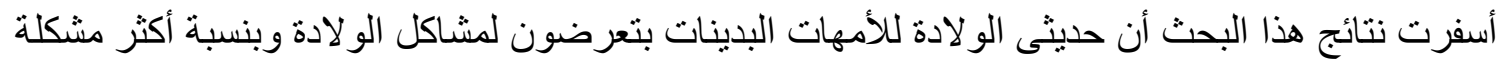

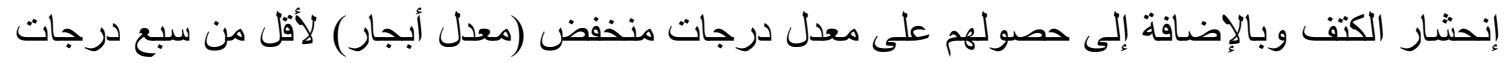

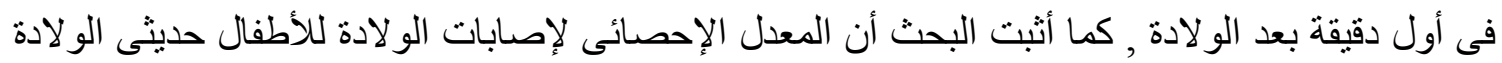

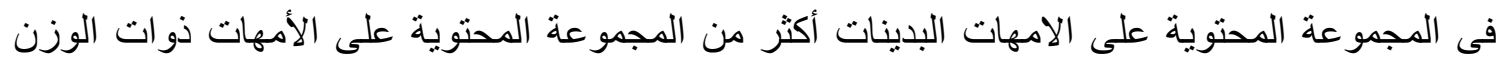

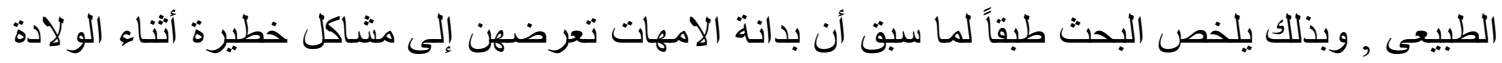

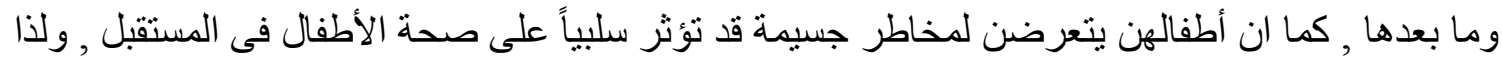

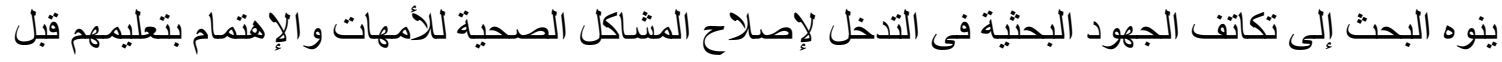
القدوم على حمل فى المستقبل . 